\title{
Point-Prevalence Survey for the Hospital-Acquired Infections in Intensive Care Units of Trauma Center in a Tertiary Care Hospital of Northern India
}

\author{
Sandeep Boora ${ }^{1}$ Parul Singh ${ }^{2}$ Arun Verma ${ }^{1}$ Ashok Chauhan ${ }^{1}$ Amit Lathwal ${ }^{1}$ Purva Mathur $^{2}$ \\ ${ }^{1}$ Hospital Administration, JPN Apex Trauma Center, All India Institute \\ of Medical Sciences, New Delhi, India \\ 2 Departments of Microbiology, JPN Apex Trauma Center, All India \\ Institute of Medical Sciences, New Delhi, India \\ Address for correspondence Purva Mathur, MD, Department of \\ Laboratory Medicine (Microbiology), JPN Apex Trauma Center, All \\ India Institute of Medical Sciences, Room. No. 325, Sri Aurobindo \\ Marg, Ansari Nagar, New Delhi 110029, India \\ (e-mail: purvamathur@yahoo.co.in).
}

J Lab Physicians 2022;14:115-118.

\begin{abstract}
Background The burden of hospital-acquired infections (HAls) is all assumption based, and the true burden remains unknown in most countries, particularly in the developing countries where healthcare facilities are suboptimal and knowledge is limited.

Methodology This cross-sectional study was conducted at the trauma center of a tertiary care institute from August to September 2019, to assess the burden of HAI and antibiotic resistance pattern of HAl. The total sample size in our study was 105 . Our objective was to estimate the point prevalence of HAI and study the associated factors in a tertiary care hospital.

Result In this study, the point prevalence of HAI was five to six times higher when compared with that of developed countries. Gram-negative organisms were the predominant bacteria; with Acinetobacter baumannii the most common among them.

Keywords

- hospital-acquired infection

- point prevalence

- Acinetobacter baumannii

Conclusion Point-prevalence survey is an important objective of the antimicrobial stewardship program; it will be helpful in controlling antimicrobial resistance and this tool plays a significant role in hospital settings. Our study is quite pertinent to assess the point prevalence of HAl. It will help in knowing the current prevalence and pattern of the HAI. Therefore, as healthcare administrators, we can further decrease the HAl for better patient outcomes in the future.
\end{abstract}

\section{Introduction}

Hospital-acquired infections (HAI) are defined as "infections occurring in a patient or in a hospital or another healthcare facility in whom the infection was not present or incubating at the time of admission."1

HAIs are associated with increased mortality and morbidity and pose a tremendous strain to the health system and it is considered as a major public health problem in developed as well as developing countries. It is also responsible for an

published online July 9, 2021
DOI https://doi.org/

10.1055/s-0041-1731946. ISSN 0974-2727. increase in the average length of stay in hospital and increased healthcare expenditure. As per the estimates, in the United States, HAIs are associated with 88,000 deaths and an economic burden of $\$ 4.5$ billion per year. ${ }^{2-5}$

To check this problem, effective surveillance of HAIs is an important step for every healthcare facility. The surveillance mechanism not only helps in reducing the HAIs but also helps in quality improvement by identifying and rectifying the problem in the hospitals. The increasing trend in HAIs is a universal problem and every health system has a dedicated (c) 2021. The Indian Association of Laboratory Physicians. All rights reserved.

This is an open access article published by Thieme under the terms of the Creative Commons Attribution-NonDerivative-NonCommercial-License, permitting copying and reproduction so long as the original work is given appropriate credit. Contents may not be used for commercial purposes, or adapted, remixed, transformed or built upon. (https://creativecommons.org/ licenses/by-nc-nd/4.0/)

Thieme Medical and Scientific Publishers Pvt. Ltd., A-12, 2nd Floor, Sector 2, Noida-201301 UP, India 
surveillance team/mechanism to control the HAI. The surveillance mechanism can be prospective or retrospective and each method has its advantages and disadvantages. ${ }^{6}$

As per the Centers for Disease Control and Prevention (CDC), "The gold standard for HAI surveillance is a large, prospective, continuous, hospital-wide, incidence survey. However, few hospitals opt for this approach because incidence surveys require a significant amount of manpower, material resources and a greater number of trained personnel., ${ }^{-9}-9$

In world history, there have been many diseases that humans have had to fight. We have used natural remedies, antibiotics, surgical procedures, and vaccines to treat and prevent the disease from occurring or spreading. Our focus on medicine and treatment of disease from history to today is due to our burning desire to keep human beings alive and well for as long as we can. To study our progression with the treatment of disease, we must use point prevalence.

Point prevalence is defined as "the number of people who have a disease over the total amount of people in the population at a particular point in time. If we can pinpoint how many people are suffering from a disease at one time, then we can see if that number has increased or decreased the next time that is how, we find the point prevalence. We can also see the need for further research or funding to treat a disease." 6

Point-prevalence surveys are simple, fast, and relatively inexpensive compared with incidence surveys. ${ }^{9}$ The prevalence of HAIs varies with the socioeconomic level, the type of medical facilities, ethnicity, dietary habits, lifestyle, and behaviors. ${ }^{10,11}$ It's an important objective of antimicrobial stewardship program and will help in controlling antimicrobial resistance (AMR).

In most of the countries, the surveillance criteria have been incorporated with the definitions and diagnostic criteria for "healthcare-associated surveillance" established by the CDC. Despite difference in the diagnostic criteria of HAI, it is observed from various studies that the prevalence of HAI is inversely related to national prosperity. ${ }^{2,3}$

AMR is another challenge that is closely related to HAIs. Inappropriate use of antimicrobials is the most important cause of the emergence and spread of resistant microorganisms at the community and hospitals.

Conducting continuous active surveillance helps to identify the root causes of HAIs, patterns of microorganisms, and types of antibiotic resistance that help the infection control team of a hospital to improve their management protocols. Additionally, point-prevalence surveys of HAIs are an important tool for comparison among the healthcare facilities at national as well as at the international level. ${ }^{1}$

\section{Need of the Study}

In most of the cases, the burden of HAIs is all assumption based, and the true burden remains unknown in most of the countries, particularly in the poor countries where healthcare facilities are suboptimal. Addition to this, limited data are available from India. Developing nations like India, where advance technology to monitor adherence may not be available, direct observation remains the choice. Continuous training, performance feedback, verbal reminders and effective surveillance will be needed to sustain adherence to prevent and control HAI. So, our study is quite pertinent to assess the point prevalence of HAI; it will help to know the current prevalence and pattern of the HAI. Therefore, as a healthcare administrator, we can further decrease the HAI for better patient outcomes in the future.

\section{Methodology}

This cross-sectional study was conducted on the trauma center, of a tertiary care institute from August to September 2019, to assess the burden of HAI and antibiotic resistance pattern of HAI. For the data collection, by using a convenient sampling method, the existing HAI checklist was used by the investigator. Our study population includes all the patients of polytrauma intensive care unit (ICU) who were initiated on parenteral antibiotics during the study period of 24 hours. The case sheets of these patients are obtained from the ICUs to determine if appropriate cultures (blood, respiratory secretions, urine, pus, or cerebrospinal fluid) have been sent for these patients, before initiating or changing antibiotics, for dosage adjustments. Total sample in our study was 105. Data were analyzed for frequencies, percentages, and mean value that were calculated using the Statistical Package of Social Sciences (SPSS), Version22. The aim of our study was to estimate the point prevalence of HAI and study the associated factors in a tertiary care hospital.

\section{Results}

Total sample size was 105 . Mean age of the patients was $38.70 \pm 20.51$ years. Majority of patients were male 82 (78.1\%). Our study revealed that most of the admitted patients were suffering from polytrauma (43; $41 \%)$, followed by abdominal injury (22; $21 \%)$, long bone injury (22; $21 \%$ ), chest injury (13; 12.4\%), spinal injury $(3 ; 12.4 \%)$, and neck and vascular injury (1 each; $1 \%)$. It was observed that empirical treatment was started for 64 (61\%) patients. Additionally, for the rest of the patients, the sample was taken for microbiological culture and sensitivity test. No growth was observed in 73 patients. The prevalence of HAI was observed at $30.4 \%$. The most common sample taken was bronchoalveolar lavage (BAL) 15 (14.3\%) and blood 15 (14.3\%), followed by pus $6(5.7 \%)$, tissue $3(2.9 \%)$, wound swab $1(1 \%)$, and others body fluids $1(1 \%)$. After getting the culture report, we found that Acinetobacter baumannii was the most common organism (9; 8.6\%), followed by Escherichia coli ( $6 ; 6.2 \%)$ and Klebsiella spp. (5; 5.2\%). However, Pseudomonas spp., Proteus spp., Stenotrophomonas maltophilia, and Enterococcus spp. constitute $1 \%$ each from the culture. Initially, most 44 (41.9\%) of the patients were put into two antibiotics, followed by 23 patients (21.9\%) who were put on three antibiotics, 17 (16.2\%) on four antibiotics, and 16 (15.2\%) into one antibiotic. However, it was noted that only one patient was put on seven antibiotic combinations. It was also observed that 
Table 1 Variables pertaining to the point-prevalence study

\begin{tabular}{|c|c|c|}
\hline Variables & $n$ & $\%$ \\
\hline \multicolumn{3}{|l|}{ Sex } \\
\hline Female & 23 & 21.9 \\
\hline Male & 82 & 78.1 \\
\hline \multicolumn{3}{|l|}{ Types of trauma } \\
\hline Polytrauma & 43 & 41 \\
\hline Abdomen injury & 22 & 21 \\
\hline Chest injury & 13 & 12.4 \\
\hline Spinal injury & 3 & 2.9 \\
\hline Neck injury & 1 & 1 \\
\hline Vascular injury & 1 & 1 \\
\hline \multicolumn{3}{|l|}{ Microorganisms } \\
\hline Acinetobacter baumannii & 9 & 8.6 \\
\hline Escherichia coli & 6 & 6.2 \\
\hline Klebsiella spp. & 5 & 5.2 \\
\hline Enterococcus spp. & 1 & 1 \\
\hline Enterobacter spp. & 1 & 1 \\
\hline Proteus spp. & 1 & 1 \\
\hline Pseudomonas spp. & 1 & 1 \\
\hline Stenotrophomonas maltophilia & 1 & 1 \\
\hline Providencia spp. & 1 & 1 \\
\hline \multicolumn{3}{|l|}{ Samples } \\
\hline Bronchoalveolar lavage & 15 & 14.3 \\
\hline Blood & 15 & 14.3 \\
\hline Pus & 6 & 5.7 \\
\hline Tissue & 3 & 2.9 \\
\hline Wound swab & 1 & 1.0 \\
\hline Fluid & 1 & 1.0 \\
\hline \multicolumn{3}{|l|}{ Total number of antibiotics used } \\
\hline 1.00 & 16 & 15.2 \\
\hline 2.00 & 44 & 41.9 \\
\hline 3.00 & 23 & 21.9 \\
\hline 4.00 & 17 & 16.2 \\
\hline 5.00 & 4 & 3.8 \\
\hline 7.00 & 1 & 1.0 \\
\hline \multicolumn{3}{|l|}{ Drugs/drug combinations given against } \\
\hline Gram negative & 60 & 63.2 \\
\hline Gram-negative and gram-positive agents & 15 & 15.8 \\
\hline Gram negative and antifungal agents & 6 & 6.3 \\
\hline Gram negative, gram positive, and antifungal agents & 6 & 6.3 \\
\hline Gram positive agents & 5 & 5.3 \\
\hline Gram positive and antifungal agents & 2 & 2.1 \\
\hline Antifungal agents & 1 & 1.1 \\
\hline
\end{tabular}

initially, a drug against gram-negative bacteria was most commonly used $(60 ; 63.2 \%)$, followed by gram-positive and gram-negative combination 15 (15.8\%); gram-positive, gram-negative, and antifungals were used in combination among six (6.3\%) patients. Only gram-positive drug was administered for five patients (5.3\%, - Table $\mathbf{1})$.

\section{Discussion}

Our pilot study was aimed to estimate the point prevalence of HAI and study the associated risk factors in a tertiary care hospital. The point prevalence came around $30.4 \%$, which is quite high as compared with mean HAI prevalence of $7.1 \%$ 
reported by the European Centre for Disease Prevention and Control and 6.4\% reported by the English National Point Prevalence Survey on HAI. However, a similar finding was observed in a study conducted by Shin et al where point prevalence came around $33.5 \%{ }^{12}$ In our study, it was observed that most of our patients were male; similar findings were observed in a study conducted by Hassan and El-Gilany in Egypt. ${ }^{13}$ This may be because we conducted this study in a trauma center and men are involved more in outdoor activities; so they are more prone to accidents. A similar finding was observed from the Global Burden of Disease Study ${ }^{14}$ in which patients were mostly middle-aged. The most common sample taken was BAL and blood, followed by pus, tissue, wound swab, and other body fluids. This may be because most of our patients suffered from multiple traumas and, due to technical feasibility, the most common samples were blood and BAL.

Regarding culture results, the predominant organisms were gram-negative and similar findings were observed in a study conducted by Shin et al. ${ }^{12}$ Among gram-negative organisms, A. baumannii (a gram-negative rod) was the most common organism found among the patients, followed by $E$. coli and Klebsiella spp. Similar findings were observed in a study in India conducted by Choudhuri et al. ${ }^{15}$ Our study also revealed that most offered initial modality of treatment for the trauma patients was a combination of two antibiotics, followed by three and four antibiotics, respectively.

It was also observed that after the culture report, a drug against gram-negative bacteria were mostly used, followed by gram-positive and gram-negative combination. This finding suggests that HAI awareness and education are well covered in our ICU settings and use of antibiotics among trauma patients providing them prompt gram-negative coverage based on culture reports is prescribed.

To summarize, our study indicated that polytrauma patients were more commonly admitted in the trauma center; they are more prone to HAI by gram-negative organisms. The prevalence of HAI in our study was five to six times higher compared with the developed countries as they have fewer chances of infections. As a hospital administrator, there is an urgent need to reduce the point prevalence of HAI by proper training and interdepartmental collaboration, where the Hospital Infection Control Committee should play a leadership role.

\section{Limitation of the Study}

We conducted this study in a small period with a single area of a trauma center, so we have less sample size. Therefore, it is not possible to generalize our findings. To do that in future, we need a larger sample size with a longer period.

We also need to consider the associated risk factors that we did not take into the account in our study due to feasibility issues.

\section{Conclusion}

The point prevalence of HAI in our study was five to six times higher compared with the developed countries. It has been observed that gram-negative organisms were predominant for causing HAI. Among them A. baumannii was the most common gram-negative organism that was reported to cause infections. Drugs targeted on gram-negative organisms are most used antibacterial agents among the HAI patients based on the culture reports. Therefore, carrying out culture of cultures will be helpful in antimicrobial stewardship programs and controlling AMR.

\section{Conflict of Interest}

None reported.

\section{References}

1 Raka L, Spahija G, Gashi-Gecaj Aet al.Point prevalence survey of healthcare-associated infections and antimicrobial use in Kosovo hospitals. Infect Dis Rep 2019;11(01):7975 Doi: 10.4081/ idr.2019.7975

2 Nair V, Sahni AK, Sharma Det al.Point prevalence \& risk factor assessment for hospital-acquired infections in a tertiary care hospital in Pune, India. Indian J Med Res 2017;145(06):824-832

3 Haque M, Sartelli M, McKimm J, Abu Bakar M. Health careassociated infections - an overview. Infect Drug Resist 2018; 11:2321-2333

4 Stone PW. Economic burden of healthcare-associated infections: an American perspective. Expert Rev Pharmacoecon Outcomes Res 2009;9(05):417-422

5 HAI Data | CDC. Available at: https://www.cdc.gov/hai/data/index. html [cited May 29, 2019]. Accessed May 29, 2021

6 Liu JY, Wu YH, Cai M, Zhou CL. Point-prevalence survey of healthcare-associated infections in Beijing, China: a survey and analysis in 2014. J Hosp Infect 2016;93(03):271-279

7 Yallew WW, Kumie A, Yehuala FM. Point prevalence of hospitalacquired infections in two teaching hospitals of Amhara region in Ethiopia. Drug Healthc Patient Saf 2016;8:71-76

8 Askarian M, Yadollahi M, Assadian O. Point prevalence and risk factors of hospital acquired infections in a cluster of universityaffiliated hospitals in Shiraz, Iran. J Infect Public Health 2012; 5(02):169-176

9 Malobicka E, Roskova D, Svihrova V, Hudeckova H. Point prevalence survey of nosocomial infections in university hospital in Martin. Acta Medica Martiniana. 2013;13(02):34-41

10 WHO.The burden of health care-associated infection worldwide. Available at: https://www.who.int/gpsc/country_work/burden_hcai/en/ [cited Jan 16, 2019]. Accessed May 29, 2021

11 Current HAI Progress Report | CDC. Available at: https://www. cdc.gov/hai/data/portal/progress-report.html [cited May 29, 2019]. Accessed May 29, 2021

12 Shin YM, Oh YM, Kim MNet al.Usefulness of quantitative endotracheal aspirate cultures in intensive care unit patients with suspected pneumonia. J Korean Med Sci 2011;26(07):865-869

13 Hassan R, El-Gilany A-H, Amina M, Abd elaal, Noha El-Mashad, Dalia Abdel Azim. An overview of healthcare-associated infections in a tertiary care hospital in Egypt Infection Prevention in Practice 23 100059. . Doi: 10.1016/j.infpip.2020.100059

14 GBD 2019 Diseases and Injuries Collaborators. Global burden of 369 diseases and injuries in 204 countries and territories, 19902019: a systematic analysis for the Global Burden of Disease Study 2019. Lancet 2020;396(10258): 1204-1222. DOI: 10.1016/ S0140-6736(20)30925-9. Erratum in: Lancet. 2020 Nov 14;396 (10262):1562. PMID: 33069326; PMCID: PMC7567026

15 Choudhuri AH, Chakravarty M, Uppal R. Epidemiology and characteristics of nosocomial infections in critically ill patients in a tertiary care intensive care unit of Northern India. Saudi J Anaesth 2017;11(04):402-407 